\title{
Near-field observation of subwavelength confinement of photoluminescence by a photonic crystal microcavity
}

\author{
Nicolas Louvion, Adel Rahmani, Christian Seassal, and Ségolène Callard \\ Laboratoire d'Elecronique, Optoélectronique et Microsystèmes, UMR CNRS 5512, Ecole Centrale de Lyon, \\ 36 Avenue Guy de Collongue, F-69134 Ecully Cedex, France \\ Davy Gérard* and Frédérique de Fornel \\ Laboratoire de Physique de l'Université de Bourgogne, CNRS UMR 5027, Boîte Postale 47870 F-21078, \\ Dijon Cedex, France
}

\begin{abstract}
Received February 14, 2006; revised April 24, 2006; accepted May 7, 2006; posted May 10, 2006 (Doc. ID 68093) We present a direct, room-temperature near-field optical study of light confinement by a subwavelength defect microcavity in a photonic crystal slab containing quantum-well sources. The observations are compared with three-dimensional finite-difference time-domain calculations, and excellent agreement is found. Moreover, we use a subwavelength cavity to study the influence of a near-field probe on the imaging of localized optical modes. (C) 2006 Optical Society of America
\end{abstract}

OCIS codes: $230.0230,180.5810,250.5230$.

By combining high-refractive-index contrast and lattice effects, photonic crystals (PCs) have demonstrated an unprecedented ability to control and confine photons. 1 Even structures exhibiting an incomplete bandgap, which prevent the propagation of photons within a range of frequencies only in some directions, have led to an impressive array of new devices. In particular, slablike structures that rely on total internal reflection for vertical optical confinement while the photonic bandgap effect controls the in-plane propagation play an increasingly important role in nanophotonics. When combined with defect engineering, such structures can support localized modes with small mode volumes and large quality factors. ${ }^{2}$ Coupled to a single emitter, these PC structures can be used to enhance or inhibit the dynamics of the source ${ }^{3-5}$ or even as a source of nonclassical light. ${ }^{6}$ Strong coupling between a quantum dot and a two-dimensional PC slab cavity has also been reported. ${ }^{7}$

The efficiency of the coupling between an emitter and a cavity depends both on the spectral tuning and the spatial overlap between the cavity mode and the emitter. To address the issue of optimal positioning of an emitter inside a cavity it is crucial to know the actual cavity mode profile on a small enough scale. For that purpose, near-field scanning optical microscopy (NSOM) has proved to be an invaluable tool. ${ }^{8-14}$ However, before dealing with more involved structures, it is important to study carefully some basic structures for which the influence of the near-field probe on the observed mode profile can be assessed.

In this Letter, we present a NSOM study of the confinement of the photoluminescence (PL) of quantum wells (QWs) by a subwavelength PC microcavity. We use a NSOM stand-alone system ${ }^{15}$ in collection mode. The near-field probe is an uncoated, chemically etched monomode optical fiber. Compared with metal-coated probes, this approach ensures that the near-field probe does not perturb significantly the optical modes of the cavity. The PC structures are optically pumped at $780 \mathrm{~nm}$ with a laser diode modulated at $2 \mathrm{kHz}$. The pump beam is focused on the structure with a long work distance objective. Because of the angle of incidence, the pump spot at the surface of the crystal is elliptic with an approximate size of $7 \mu \mathrm{m} \times 9 \mu \mathrm{m}$. The PL signal is collected by the nearfield probe and guided to a monochromator. A longpass filter is used to eliminate the pump (cutoff wavelength at $1200 \mathrm{~nm}$ ). The signal is detected with a thermo-electrically cooled InGaAs photodetector. A lock-in amplifier is used to demodulate the signal and enhance the signal-to-noise ratio. The fiber is attached to a piezoelectric tuning fork setup that allows a shear-force feedback loop to regulate the distance between the tip and the sample. In this configuration, topographic and PL images are recorded simultaneously.

The photonic crystal microcavities are fabricated on an InP slab, wafer bonded onto a $1 \mu \mathrm{m}$ thick $\mathrm{SiO}_{2}$ layer on top of a silicon host substrate. A triangular lattice of air holes is drilled using e-beam lithography and reactive ion etching. ${ }^{16}$ The $\mathrm{InP}$ slab is $250 \mathrm{~nm}$ thick, has a refractive index of 3.17 at $1.55 \mu \mathrm{m}$, and supports only one guided mode around that wavelength. Four InAsP QWs, separated by InP barrier layers, are embedded at the center of the InP slab. The PL from the QWs occurs between 1250 and $1650 \mathrm{~nm}$. The cavity is formed by introducing a single defect (omitting 1 hole) into the PC [Fig. 1(a)]. This defect will be referred to as microcavity H1.

Figure 1(a) shows a topographic image of microcavity $\mathrm{H} 1$ recorded by shear-force microscopy. The microcavity is clearly visible. The period of the crystal is $540 \mathrm{~nm}$, and the radius of the holes is $210 \mathrm{~nm}$. H1 microcavities support two degenerated modes. In Fig. 


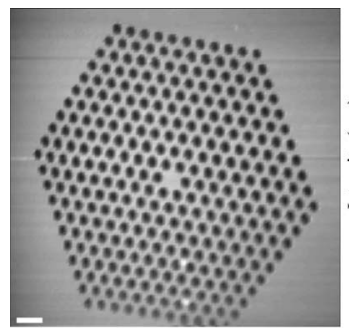

(a)

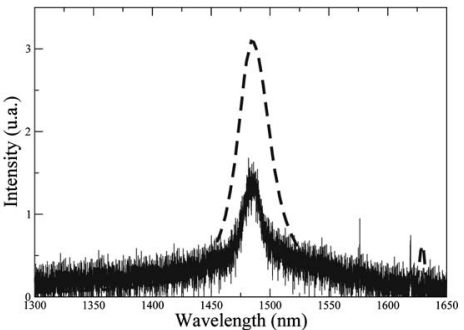

(b)
Fig. 1. (a) Shear-force topography. (b) Near-field PL spectrum (dashed curve) recorded above the cavity and far-field PL spectrum (solid curve). The bar length is $1 \mu \mathrm{m}$.
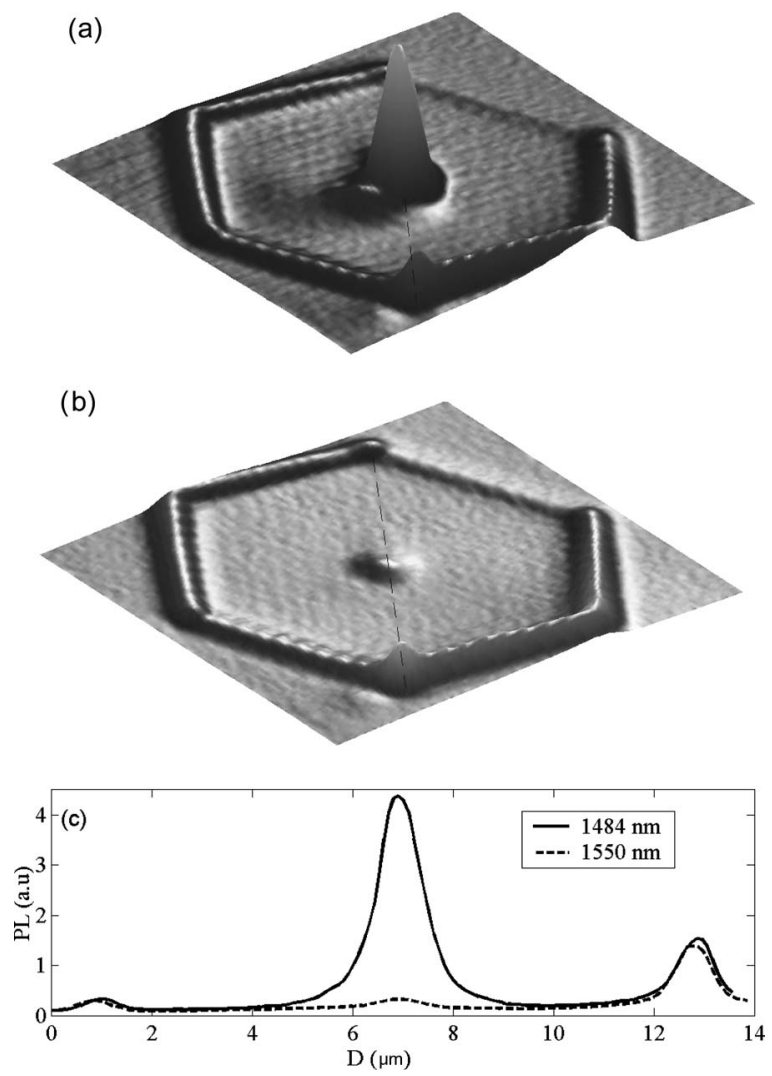

Fig. 2. Experimental near-field map of the cavity mode at (a) $1484 \mathrm{~nm}$ and (b) $1550 \mathrm{~nm}$. (c) Vertical near-field intensity profiles at $1484 \mathrm{~nm}$ (solid curve) and $1550 \mathrm{~nm}$ (dashed curve).

1(b) we plot the PL spectrum recorded in the near field with the tip at the center of the cavity (dashed curve). The PL spectrum recorded with a conventional far-field setup is also shown (solid curve). The two independent spectral measurements agree perfectly on the wavelength of the resonance $(1484 \mathrm{~nm})$, thereby illustrating that the probe does not induce any shift of the cavity resonance.

Figures 2(a) and 2(b) are two PL near-field maps of the entire structure recorded at 1484 and $1550 \mathrm{~nm}$, respectively. Figure 2(a) is the near-field optical map at the cavity resonance. The peak located at the center of the structure is the recorded profile of the cavity mode. No light is collected above the holes outside the cavity, which is a clear and direct illustration of the photonic bandgap effect. Note that the PL detected at the edges of the photonic crystal, on the first row of holes, is due to QWs outside the patterned region. Since the pump beam is not confined to the crystal, PL occurs outside the crystal, couples to the slab guided mode and is scattered at what is essentially a PC mirror. This is further confirmed by a local near-field spectroscopic measurement performed above the first row of holes, which matched the broad PL spectrum of the QWs (as opposed to the much narrower spectral response of the photonic crystal mode).

Figure 2(b) shows the near-field optical map recorded away from the cavity resonance. A weak peak is observed above the cavity that corresponds to the residual $\mathrm{PL}$ emitted by the $\mathrm{QWs}$. We emphasize that this PL peak is not due to any cavity mode, rather it is merely the result of having more active material in the cavity. Figure 2(c) shows a comparison between intensity profiles at and off resonance. The curves are normalized to the QWs PL of an unpatterend area to account for variations in the $\mathrm{QW}$ emission spectrum. It shows that the experimental exaltation factor of the cavity mode is about 9.4. The calculated exaltation factor, computed from three-dimensional (3D) finite-difference time domain (FDTD) calculations, is about 25. The difference between computed and measured values can be explained by the fact that the real cavity, due to geometrical fluctuation inherent in the fabrication process, suffers larger losses than the ideal cavity. This is confirmed by the differences in quality factor between the computed value of 85 and the measured value of 55 .

Figures 3(a) and 3(b) show the topography and the near-field PL cartography (1484 $\mathrm{nm}$ ) of the same cavity. The cavity mode is slightly elliptic with dimensions of $0.92 \mu \mathrm{m} \times 1 \mu \mathrm{m}$ at full width at halfmaximum.

The 3D FDTD computation allows us to make a direct comparison between theory and the experimental near-field map of Fig. 3(b). The theory predicts two dipole modes with orthogonal polarization. As they are degenerate and are evenly excited, these modes contribute equally to the mode pattern. The computed cavity modes are found at $1498 \mathrm{~nm}$, and the intensity map computed at the slab surface is shown in Fig. 4(a). The discrepancy between the experimental wavelength $(1484 \mathrm{~nm})$ and the computed one $(1498 \mathrm{~nm})$ is less that $1 \%$ and comes mainly from the fact that the geometric fluctuations that are inherent in the fabrication of the structure cannot be accounted for in the FDTD calculation. Compared with the measurement, the computed map shows finer details: the cavity mode has six bright lobes lo-
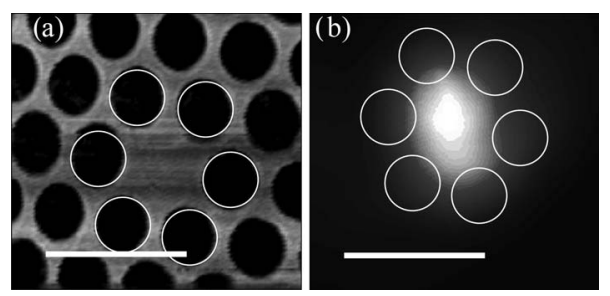

Fig. 3. (a) Shear-force topography. (b) Experimental nearfield map of the degenerate mode at $1484 \mathrm{~nm}$. The bar length is $1 \mu \mathrm{m}$. 

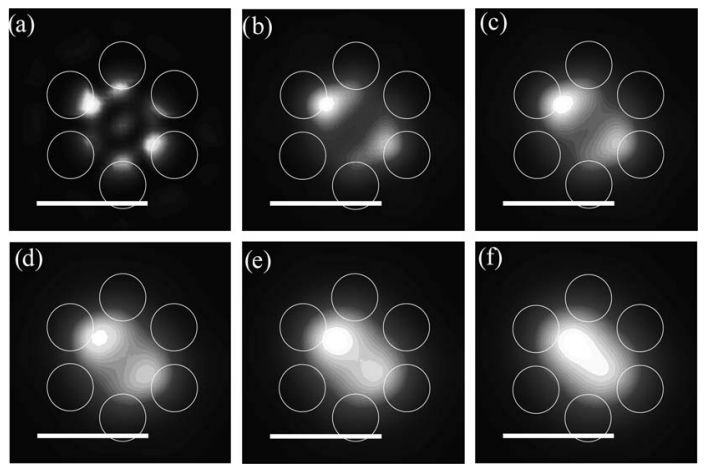

Fig. 4. (a) Map of the degenerate modes at $1498 \mathrm{~nm}$ computed by 3D FDTD at the surface of the membrane, computed maps filtered with a Gaussian filter with (b) $\sigma$ $=125 \mathrm{~nm}$, (c) $\sigma=150 \mathrm{~nm}$, (d) $\sigma=175 \mathrm{~nm}$, (e) $\sigma=200 \mathrm{~nm}$, and (f) $\sigma=225 \mathrm{~nm}$. The bar length is $1 \mu \mathrm{m}$.

cated at the edges of the holes and a weaker peak at the center of the cavity. The size of the lobes is about $100 \mathrm{~nm}(\lambda / 15)$. These lobes are not resolved by the near-field setup because the apex of the probe has a physical diameter of about $200 \mathrm{~nm}$. Furthermore, the collection cross section of the tip exceeds its physical apex section, as the coupling process for an uncoated fiber tip involves a finite region above the apex. As a matter of fact, we found that the $\mathrm{H} 1$ microcavity was a good reference from which to estimate the actual collection cross section of the tip. To take the finite size of the tip into account in the simulation, we filtered the computed map in Fig. 4(a) with a Gaussian filter whose standard deviation $\sigma$ was varied from 125 to $250 \mathrm{~nm}$ in $25 \mathrm{~nm}$ increments. The best agreement with the measurement was found for $\sigma$ $=200 \mathrm{~nm}$ [Fig. 4(e)]. For other probes, $\sigma$ can be as low as $175 \mathrm{~nm}$. For near-infrared applications $(\lambda$ $=1.5 \mu \mathrm{m}$ ), a spatial resolution of $470 \mathrm{~nm}$ (FWHM of the Gaussian filter) translates into $0.3 \lambda$, which is better than the diffraction limit $(0.5 \lambda)$. It is, however, not precise enough to reveal the fine structure of the H1 mode. It would be interesting to perform a similar experiment with a metal-coated probe. However, one would first have to ensure that the probe does not alter significantly the mode profile and/or resonance.

In summary, we have observed the confined mode of an $\mathrm{H} 1$ active cavity and compared it with 3D FDTD simulations. We have observed a clear signature of the confinement of light by the subwavelength cavity at resonance. A nearly tenfold enhancement of the PL above the cavity has been observed when the cavity mode is excited. The high-contrast mode pattern observed at the resonance wavelength of the cavity has also allowed us to assess the collection cross section of an uncoated near-field probe.
We thank Philippe Regreny for growing the epitaxial structures. The wafer bonding technology was supplied by CEA-LETI. This work was supported by the Ministère Délégué à la Recherche et aux Nouvelles Technologies (Program "Action Concertée Incitative Jeune Chercheur") and the Centre National de la Recherche Scientifique (Réseau Thématique Pluridisciplinaire 29 SNOBIP). S. Callard's e-mail address is segolene.callard@ec-lyon.fr.

*Present address, Institut FEMTO-ST Départments LPMO et LOPMD, 32 Avenue de l'Observatoire F-25044 Besançon Cedex, France.

\section{References}

1. J. D. Joannopoulos, R. Meade, and J. Winn, Photonic Crystals (Princeton U. Press, 1995).

2. Y. Akahane, T. Asano, B.-S. Song, and S. Noda, J. Design Manuf. Autom. 425, 944 (2003).

3. M. Fujita, S. Takahashi, Y. Tanaka, T. Asano, and S. Noda, Science 308, 1296 (2005).

4. D. Englund, D. Fattal, E. Waks, G. Solomon, B. Zhang, T. Nakaoka, Y. Arakawa, Y. Yamamoto, and J. Vuckovic, Phys. Rev. Lett. 95, 013904 (2005).

5. K. Kounoike, M. Yamaguchi, M. Fujita, T. Asano, J. Nakanishi, and S. Noda, Electron. Lett. 41, 1402 (2005).

6. J. Vučković and Y. Yamamoto, Appl. Phys. Lett. 82, 2374 (2003).

7. T. Yoshie, A. Scherer, J. Hendrickson, G. Khitrova, H. M. Gibbs, G. Rupper, C. Ell, O. B. Shchekin, and D. G. Deppe, Nature 432, 200 (2004).

8. D. Gérard, L. Berguiga, F. de Fornel, L. Salomon, C. Seassal, X. Letartre, P. Rojo-Romeo, and P. Viktorovitch, Opt. Lett. 27, 173 (2002).

9. D.-J. Shin, S.-H. Kim, J.-K. Hwang, H.-Y. Ryu, H.-G. Park, D.-S. Song, and Y.-H. Lee, IEEE J. Quantum Electron. 38, 857 (2002).

10. E. Flück, M. Hammer, A. M. Otter, J. P. Korterik, L. Kuipers, and N. F. van Hulst, J. Lightwave Technol. 21, 1384 (2003).

11. K. Okamoto, M. Loncar, T. Yoshie, A. Scherer, Y. Qiu, and P. Gogna, Appl. Phys. Lett. 82, 1676 (2003).

12. P. Kramper, M. Agio, C. M. Soukoulis, A. Birner, F. Muller, R. B. Wehrspohn, U. Gosele, and V. Sandoghdar, Phys. Rev. Lett. 92, 113903 (2004).

13. P. Kramper, M. Kafesaki, C. M. Soukoulis, A. Birner, F. Müller, U. Gösele, R. B. Wehrspohn, J. Mlynek, and V. Sandoghdar, Opt. Lett. 29, 174 (2004).

14. N. Louvion, D. Gérard, J. Mouette, F. de Fornel, C. Seassal, X. Letartre, A. Rahmani, and S. Callard, Phys. Rev. Lett. 94, 113907 (2005).

15. A commercial SMENA-NSOM manufactured by the NTMDT company was used; http://www.ntmdt.ru/.

16. C. Monat, C. Seassal, X. Letartre, P. Regreny, P. RojoRomeo, P. Viktorovitch, M. Le Vassor d'Yerville, D. Cassagne, J. P. Albert, E. Jalaguier, S. Pocas, and B. Aspar, IEEE J. Quantum Electron. 39, 419 (2003). 